\title{
Sign-constancy of Green's functions for impulsive nonlocal boundary value problems
}

\section{A. Domoshnitsky ${ }^{1}$ and lu. Mizgireva ${ }^{1,2^{*}}$ (1)}

\section{${ }^{*}$ Correspondence:}

julia.mizgireva@gmail.com

${ }^{1}$ Department of Mathematics, Ariel University, Ariel, Israel

${ }^{2}$ Laboratory of Cryptoeconomics and Blockchain Systems, Perm State

University, Perm, Russia

\section{每 Springer}

\begin{abstract}
We consider the following second order impulsive differential equation with delays:

$$
\left\{\begin{array}{l}
(L x)(t) \equiv x^{\prime \prime}(t)+\sum_{j=1}^{p} a_{j}(t) x^{\prime}\left(t-\tau_{j}(t)\right)+\sum_{j=1}^{p} b_{j}(t) x\left(t-\theta_{j}(t)\right)=f(t), \quad t \in[0, \omega], \\
x\left(t_{k}\right)=\gamma_{k} x\left(t_{k}-0\right), \quad x^{\prime}\left(t_{k}\right)=\delta_{k} x^{\prime}\left(t_{k}-0\right), \quad k=1,2, \ldots, r .
\end{array}\right.
$$

In this paper we consider sufficient conditions of nonpositivity of Green's function for impulsive differential equation with nonlocal boundary conditions.
\end{abstract}

Keywords: Second order impulsive differential equations; Boundary value problems; Sign-constancy of Green's functions

\section{Introduction}

Impulsive differential equations have attracted an attention of a number of recognized mathematicians and have applications in many spheres of science from physics, biology, medicine to economical studies. The following well-known books can be noted in this context [35, 41, 44, 54]. In Ref. [4], the concept of the general theory of functional differential equations was presented. In the frame of this concept finite fundamental system, the Wronskian and Green's functions can be introduced. On the basis of this concept nonoscillation for the first order impulsive functional differential equations was considered in [10], where positivity of the Cauchy and Green's functions of the periodic problem was firstly studied. Nonoscillation for the first order impulsive differential equations is also considered in the book [1]. The positivity of Green's function of one- and two-point boundary value problems for impulsive functional differential equations of the first order was considered in [10] and of the second order in [2,11, 12, 14, 19, 24, 28, 29, 36, 38].

The study of nonlocal boundary value problems has its own history (see, for example, its description in [8]). Multi-point and integral boundary conditions are widely studied in the case of ordinary differential equations. Maybe the work by Picone [42], where the multi-point boundary conditions are studied, is the first one. Note the review by Whyburn [52] of 1942 on problems with the Stieltjes integral in boundary conditions. Note also the works by Ma [37], Ntouyas [39], Webb and Infante [50, 51]. The positivity of solutions to non-impulsive ordinary differential equations with nonlocal boundary conditions was studied in Refs. [32, 50, 51]. The existence of positive solutions for nonlocal functional differential boundary value problems was studied in [31] and then in [30]. For example, in

(c) The Author(s) 2019. This article is distributed under the terms of the Creative Commons Attribution 4.0 International License (http://creativecommons.org/licenses/by/4.0/), which permits unrestricted use, distribution, and reproduction in any medium, provided you give appropriate credit to the original author(s) and the source, provide a link to the Creative Commons license, and indicate if changes were made. 
[31] the nonlocal boundary value problem

$$
\begin{aligned}
& x^{\prime \prime}(t)+F(t, x(t))=0, \quad t \in[0,1], \quad x(t)=\varphi(t), \quad t \in[-\delta, 0], \\
& x(0)=0, \quad x(1)=\int_{t_{1}}^{t_{2}} x(s) d R(s), \quad t_{1}, t_{2} \in(0,1),
\end{aligned}
$$

was considered. Positivity of Green's functions for the first order impulsive functional differential equations with nonlocal boundary conditions was studied in [15-17]. Nonlocal boundary value problems for systems of impulsive functional differential equations were considered in [6]. Functional differential equations of second order with nonlocal conditions were considered in [8].

Various applications with nonlocal problems for ordinary and partial differential equations were presented in the known works by Skubachevskii [45, 46]. Ordinary differential equations with integral boundary conditions arise in the theory of turbulence [47], in the theory of Markov processes [18], in heat flow problems [22, 25, 27, 48, 49], in the study of the response of a spherical cap [3, 5, 40]. In the references in [7], one can find works on applications of nonlocal problems in modeling of thermostats, beams and suspension bridges.

Questions of representation solutions and solvability of nonlocal problems for functional differential equations were considered in $[4,23,33,34]$. The positivity of solutions for nonlocal boundary value problems for ordinary differential equations was studied in $[20,21,50,51,53]$. The method is to reduce nonlocal boundary value problems to the Hammerstein integral equation and then scrupulous analysis of Green's functions leads researchers to estimates (of the norm or spectral radii in linear case and the fact of a contraction in nonlinear one) of integral operators and conclusions about positivity of solutions. It seems that some of these results can be generalized also on particular cases of delay or functional differential equations, where Green's functions of ordinary differential equations could be used [1,16, 17, 20, 21, 23]. For functional differential equations, forms of Green's functions are essentially more complicated. That is why quite a different approach was proposed for nonlocal problems with functional differential equations [1,9], where various results on positivity/negativity of Green's functions were obtained. One of the main ideas is to obtain a connection between sign-constancy of Green's functions for different problems with functional differential equations. This approach presents a basic method for analysis of the solution's positivity (see, for example, Theorem $15.3 \mathrm{in} \mathrm{[1]).} \mathrm{The}$ main results are obtained in the form of theorems about differential inequalities. Choosing the test functions, researchers can get coefficient tests for positivity of Green's functions. Note that all these works concern positivity of solutions to nonlocal problems only for scalar differential equations and not for systems. There are almost no results on positivity of solutions of nonlocal problems in the case of systems. Among the results we can note results on the existence $[7,26,33,34,43]$ and results on the positivity of solution-vectors in [26].

In our paper, we consider the following impulsive equation:

$$
(L x)(t) \equiv x^{\prime \prime}(t)+\sum_{j=1}^{p} a_{j}(t) x^{\prime}\left(t-\tau_{j}(t)\right)+\sum_{j=1}^{p} b_{j}(t) x\left(t-\theta_{j}(t)\right)=f(t), \quad t \in[0, \omega],
$$




$$
\begin{aligned}
& x\left(t_{k}\right)=\gamma_{k} x\left(t_{k}-0\right), \quad x^{\prime}\left(t_{k}\right)=\delta_{k} x^{\prime}\left(t_{k}-0\right), \quad k=1,2, \ldots, r, \\
& 0=t_{0}<t_{1}<t_{2}<\cdots<t_{r}<t_{r+1}=\omega, \\
& x(\zeta)=0, \quad x^{\prime}(\zeta)=0, \quad \zeta<0,
\end{aligned}
$$

where $f, a_{j}, b_{j}:[0, \omega] \rightarrow \mathbb{R}$ are summable functions and $\tau_{j}, \theta_{j}:[0, \omega] \rightarrow[0,+\infty)$ are measurable functions for $j=1,2, \ldots, p, p$ and $r$ are natural numbers, $\gamma_{k}$ and $\delta_{k}$ are real positive numbers.

Let $D\left(t_{1}, t_{2}, \ldots, t_{r}\right)$ be a space of functions $x:[0, \omega] \rightarrow \mathbb{R}$ such that their derivative $x^{\prime}(t)$ is absolutely continuous on every interval $t \in\left[t_{i}, t_{i+1}\right), i=0,1, \ldots, r, x^{\prime \prime} \in L_{\infty}$, we assume also that there exist the finite limits $x\left(t_{i}-0\right)=\lim _{t \rightarrow t_{i}^{-}} x(t)$ and $x^{\prime}\left(t_{i}-0\right)=\lim _{t \rightarrow t_{i}^{-}} x^{\prime}(t)$ and condition (1.3) is satisfied at points $t_{i}(i=0,1, \ldots, r)$. As a solution $x$ we understand a function $x \in D\left(t_{1}, t_{2}, \ldots, t_{r}\right)$ satisfying (1.2)-(1.4).

In this paper we obtain new results on sign-constancy of Green's functions of nonlocal boundary value problems. Comparing our results with [25-27, 50, 51], we study an essentially more general object: impulsive differential equations with delays. Note that combining the approach of [25-27] and our results, nonlinear nonlocal impulsive functional differential boundary value problems can be considered in future research. Thus, the technique we proposed in this paper opens new opportunities in the study of positivity/negativity of solutions for a wide class of impulsive functional differential equations.

\section{Preliminaries}

The general solution of (1.2)-(1.4) can be represented in the form [10]

$$
x(t)=v_{1}(t) x(0)+C(t, 0) x^{\prime}(0)+\int_{0}^{t} C(t, s) f(s) d s,
$$

where

- $v_{1}(t)$ is a solution of the homogeneous equation

$$
\begin{aligned}
& (L x)(t) \equiv x^{\prime \prime}(t)+\sum_{j=1}^{p} a_{j}(t) x^{\prime}\left(t-\tau_{j}(t)\right)+\sum_{j=1}^{p} b_{j}(t) x\left(t-\theta_{j}(t)\right)=0, \quad t \in[0, \omega], \\
& x\left(t_{k}\right)=\gamma_{k} x\left(t_{k}-0\right), \quad x^{\prime}\left(t_{k}\right)=\delta_{k} x^{\prime}\left(t_{k}-0\right), \quad k=1,2, \ldots, r, \\
& 0=t_{0}<t_{1}<t_{2}<\cdots<t_{r}<t_{r+1}=\omega, \\
& x(\zeta)=0, \quad x^{\prime}(\zeta)=0, \quad \zeta<0
\end{aligned}
$$

with the initial conditions $x(0)=1, x^{\prime}(0)=0$.

- $C(t, s)$, called the Cauchy function of (2.2)-(2.4), is the solution of the equation

$$
\begin{aligned}
& \left(L_{s} x\right)(t) \equiv x^{\prime \prime}(t)+\sum_{j=1}^{p} a_{j}(t) x^{\prime}\left(t-\tau_{j}(t)\right)+\sum_{j=1}^{p} b_{j}(t) x\left(t-\theta_{j}(t)\right)=0, \quad t \in[s, \omega], \\
& x\left(t_{k}\right)=\gamma_{k} x\left(t_{k}-0\right), \quad x^{\prime}\left(t_{k}\right)=\delta_{k} x^{\prime}\left(t_{k}-0\right), \quad k=m, \ldots, r, \\
& 0=t_{0}<t_{1}<t_{2}<\cdots<t_{r}<t_{r+1}=\omega,
\end{aligned}
$$


where $m$ is a number, such that $t_{m-1}<s \leq t_{m}$,

$$
x(\zeta)=0, \quad x^{\prime}(\zeta)=0, \quad \zeta<s
$$

satisfying the initial conditions $C(s, s)=0, C_{t}^{\prime}(s, s)=1$ and $C(t, s)=0$ for $t<s$.

Below the following definition will be used.

Definition 2.1 We call $[0, \omega]$ a semi-nonoscillation interval of (2.2)-(2.4), if every nontrivial solution having a zero of derivative does not have a zero on this interval.

In Ref. [11] for (1.2)-(1.4) the following assertion was proven.

\section{Lemma 2.1 If}

(1) $b_{j}(t) \leq 0$ for $t \in[0, \omega]$.

(2) The Cauchy function $C_{1}(t, s)$ of the first order impulsive equation

$$
\begin{aligned}
& y^{\prime}(t)+\sum_{j=1}^{p} a_{j}(t) y\left(t-\tau_{j}(t)\right)=0, \quad t \in[0, \omega], \\
& y\left(t_{k}\right)=\delta_{k} y\left(t_{k}-0\right), \quad k=1,2, \ldots, r, \\
& y(\zeta)=0, \quad \zeta<0,
\end{aligned}
$$

is positive for $0 \leq s \leq t \leq \omega$.

Then the Cauchy function $C(t, s)$ of the second order impulsive equation (1.2)-(1.4) and its derivative $C_{t}^{\prime}(t, s)$ are positive in $0 \leq s \leq t \leq \omega$.

In the lemma below, we formulate the results of [10] on the conditions of positivity of Cauchy function of the first order impulsive differential equation

$$
\begin{aligned}
& y^{\prime}(t)+a_{1}(t) y\left(t-\tau_{1}(t)\right)=0, \quad t \in[0, \omega], \\
& y\left(t_{k}\right)=\delta_{k} y\left(t_{k}-0\right), \quad k=1,2, \ldots, r, \\
& y(\zeta)=0, \quad \zeta<0 .
\end{aligned}
$$

Lemma 2.2 Let $0<\delta_{j} \leq 1$ for $j=1, \ldots, r$ and the following inequality be fulfilled:

$$
\frac{1+\ln B(t)}{e} \geq \int_{m(t)}^{t} a_{+}(s) d s,
$$

where $B(t)=\prod_{j \in D_{t}} \delta_{j}, D_{t}=\left\{i: t_{i} \in\left[t-\tau_{1}(t), t\right]\right\}, a_{+}(t)=\max \left\{a_{1}(t), 0\right\}$ and $m(t)=\max \{t-$ $\left.\tau_{1}(t), 0\right\}$. Then the Cauchy function of the first order impulsive differential equation (2.9) is positive in $0 \leq s \leq t \leq \omega$.

In the case when the number of terms with delays $p>1$ the following sufficient condition of nonnegativity of the Cauchy function $C_{1}(t, s)$, proven in [10], can be used. 
Lemma 2.3 Let $a_{j}(t) \geq 0$ for $j=1, \ldots, p, 0<\delta_{k} \leq 1$ for $k=1, \ldots, r$ and

$$
\int_{0}^{\omega} \sum_{j=1}^{p} a_{j}(s) d s<\prod_{k=1}^{r} \delta_{k}
$$

then the Cauchy function of the first order impulsive differential equation (2.8) is positive in $0 \leq s \leq t \leq \omega$.

\section{Sign-constancy of Green's function for nonlocal boundary value problem in the case of $b_{j}(t) \leq 0$}

For (1.2)-(1.4) we consider the following nonlocal boundary conditions:

$$
x(0)=0, \quad x(\omega)=l x,
$$

where $l: D\left(t_{1}, t_{2}, \ldots, t_{r}\right) \rightarrow \mathbb{R}^{1}$ is a linear bounded vector-functional.

Let us consider the following particular case of boundary conditions:

$$
x(0)=0, \quad x(\omega)=\int_{0}^{\omega} A(s) x(s) d s .
$$

If the boundary value problem (1.2)-(1.4), (3.2) is uniquely solvable, then its solution can be represented as

$$
x(t)=\int_{0}^{\omega} G_{1}(t, s) f(s) d s
$$

where $G_{1}(t, s)$ is Green's function of problem (1.2)-(1.4), (3.2).

Using general representation of the solution, the following formula for Green's function can be written:

$$
G_{1}(t, s)=C(t, s)-C(t, 0) \frac{\int_{s}^{\omega} A(r) C(r, s) d r-C(\omega, s)}{\int_{0}^{\omega} A(r) C(r, 0) d r-C(\omega, 0)}
$$

For the boundary value problem (1.2)-(1.4), (3.2) the following lemma can be obtained.

Lemma 3.1 If the conditions (1) and (2) of Lemma 2.1 are fulfilled and $A(t)$ for $t \in[0, \omega]$ satisfies the conditions

$$
A(t) \geq 0, \quad \int_{0}^{\omega} A(t) d t<1
$$

and $\gamma_{k} \geq 1, \delta_{k} \geq 0, k=1,2, \ldots, r$, then the Green's function $G_{1}(t, s)$ exists and there exists an interval $\left(0, \epsilon_{s}\right)$, such that $G_{1}(t, s)<0$ for $t \in\left(0, \epsilon_{s}\right)$.

Proof It is clear that

$$
G_{1}(0, s)=-C(0,0) \frac{\int_{s}^{\omega} A(r) C(r, s) d r-C(\omega, s)}{\int_{0}^{\omega} A(r) C(r, 0) d r-C(\omega, 0)}=0 .
$$


According to Lemma 2.1, $C(t, s) \geq 0$, then

$$
\begin{aligned}
G_{1}^{\prime}(0, s) & =-C_{t}^{\prime}(0,0) \frac{\int_{s}^{\omega} A(r) C(r, s) d r-C(\omega, s)}{\int_{0}^{\omega} A(r) C(r, 0) d r-C(\omega, 0)} \\
& =-\frac{\int_{s}^{\omega} A(r) C(r, s) d r-C(\omega, s)}{\int_{0}^{\omega} A(r) C(r, 0) d r-C(\omega, 0)}<0 .
\end{aligned}
$$

This means that there exists an interval $\left(0, \epsilon_{s}\right)$, such that $G_{1}(t, s)<0$ for $t \in\left(0, \epsilon_{s}\right)$.

Remark 3.1 Let us assume that the functional $l$ is positive, i.e. $l x \geq 0$ if $x \geq 0$, and can be presented in the form of Stieltjes integral $l x=\int_{0}^{\omega} x(s) d R(s)$. In this case, a generalization of Lemma 3.1 can be obtained by repeating the proof for problem (1.2)-(1.4), (3.1). For this case, instead of the condition (3.5), the following ones can be used:

$$
l: D\left(t_{1}, t_{2}, \ldots, t_{r}\right) \rightarrow \mathbb{R}^{1} \text { is positive functional and }\|l\|<1 .
$$

Theorem 3.1 Assume that the following conditions are fulfilled:

(1) $a_{j}(t) \geq 0, b_{j}(t) \leq 0$ for $t \in[0, \omega]$.

(2) The Wronskian $W(t)$ of the fundamental system of solutions of a homogeneous equation (2.2)-(2.4) satisfies $W(t) \neq 0, t \in[0, \omega]$.

(3) The Cauchy function $C_{1}(t, s)$ of the first order equation (2.8) is positive for $0 \leq s \leq t \leq \omega$.

(4) A(t) satisfies (3.5) for $t \in[0, \omega]$.

(5) $\gamma_{k} \geq 1, \delta_{k} \geq 0, k=1,2, \ldots, r$.

Then $G_{1}(t, s) \leq 0$ for $t, s \in[0, \omega]$.

In order to prove Theorem 3.1, we will need the following lemmas from [14].

Lemma 3.2 Assume that the following conditions are fulfilled:

(1) $a_{j}(t) \geq 0, b_{j}(t) \leq 0, j=1, \ldots, p, t \in[0, \omega]$.

(2) The Wronskian $W(t)$ of the fundamental system of solutions of homogeneous equation (2.2)-(2.4) satisfies the inequality $W(t) \neq 0, t \in[0, \omega]$.

(3) The Cauchy function $C_{1}(t, s)$ of the first order equation (2.8) is positive for $0 \leq s \leq t \leq \omega$.

Then the interval $[0, \omega]$ is a semi-nonoscillation interval of (2.2)-(2.4).

Lemma 3.3 If $a_{j}(t) \geq 0, b_{j}(t) \leq 0$, then the following assertions are equivalent:

(a) The Wronskian $W(t)$ of the fundamental system of solutions of a homogeneous equation (2.2)-(2.4) satisfies $W(t) \neq 0, t \in[0, \omega]$.

(b) The Green's function $G^{\xi}(t, s)$ with boundary conditions $x(\xi)=0, x^{\prime}(\xi)=0$ is nonnegative for $t, s \in[0, \xi]$ for every $0<\xi<\omega$.

Proof of Theorem 3.1 Let us assume that $G_{1}(t, s)$ changes its sign. This means that there exists a right-hand side $f(t) \geq 0$ such that the solution $x(t)$ changes sign (see Fig. 1). According to Lemma 3.1, $x(t)<0$ for $t$ which are close to 0 . Thus, there exist the points $\eta$ and $\xi$, such that $x(\eta)=0, x^{\prime}(\xi)=0,0<\eta<\xi<\omega$, and $x(t)>0$ for $t \in(\eta, \xi)$. 


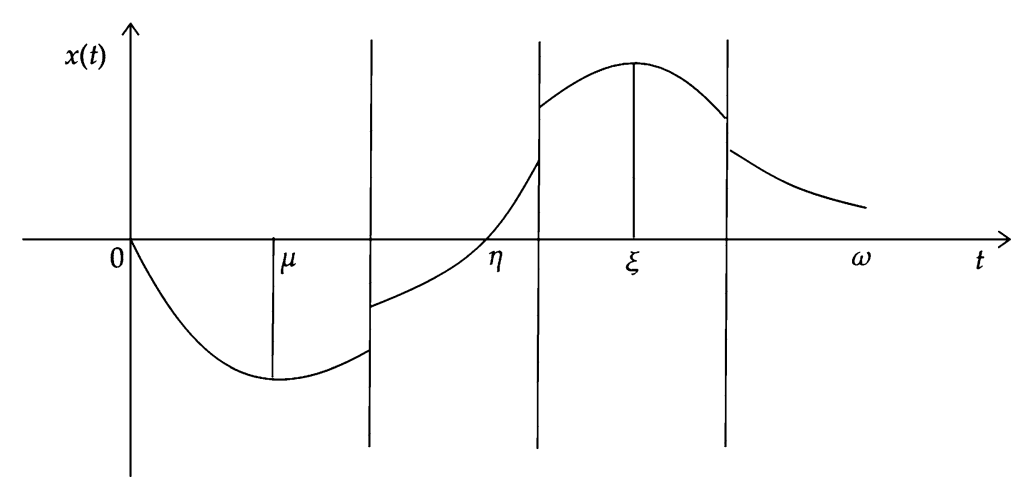

Figure 1 Solution $x(t)$ of problem (1.2)-(1.4), (3.2)

The fact of the existence of such point $\xi$ follows from the inequalities (3.5). Actually, if there is no such point $\xi$, the solution is nondecreasing for $t \in[\eta, \omega]$, since the condition (5) of Theorem 3.1 is fulfilled, and, in this case, the equality $x(\omega)=\int_{0}^{\omega} A(s) x(s) d s$ is impossible. The solution $x(t)$ satisfies the conditions

$$
x(\xi)=\alpha>0, \quad x^{\prime}(\xi)=0
$$

Consider now the problem (1.2)-(1.4) with the boundary conditions $x(\xi)=0, x^{\prime}(\xi)=0$. According to Lemma 3.3, its Green's function $G^{\xi}(t, s)$ is nonnegative for $t, s \in[0, \omega]$.

It is clear that our solution $x(t)$ of (1.2)-(1.4), (3.2) has the following representation:

$$
x(t)=\int_{0}^{\xi} G^{\xi}(t, s) f(s) d s+X(t)
$$

where $X(t)$ is the solution of the homogeneous impulsive equation (2.2)-(2.4), satisfying the conditions (3.9).

The first term $\int_{0}^{\xi} G^{\xi}(t, s) f(s) d s \geq 0$, since $G^{\xi}(t, s) \geq 0, f(s) \geq 0, t, s \in[0, \xi]$.

We see that all the conditions of Lemma 3.2 are fulfilled. This means that the interval $[0, \omega]$ is a semi-nonoscillation interval of (2.2)-(2.4). The solution $X(t)$ of homogeneous equation (2.2)-(2.4) cannot change its sign. Thus, the solution $x(t)$ is nonnegative as the sum of two nonnegative terms.

We got the contradiction with the assumption about changing sign of the solution $x(t)$. This proves that $G_{1}(t, s)$ should be nonpositive.

Remark 3.2 The conditions (2)-(3) of Theorem 3.1 look very difficult for verification, but the known previous results demonstrate that this is not the case. Lemmas 2.2-2.3 give simple inequalities implying positivity of the Cauchy function $C_{1}(t, s)$. Tests of the nonnegativity of Green's function $G^{\xi}(t, s)$ were obtained in $[12,14]$.

Remark 3.3 For boundary value problem (1.2)-(1.4), (3.1), an analogue of Theorem 3.1 can be formulated, where instead of the condition (3.5), the functional $l$ satisfies the condition (3.8). 
Example 3.1 Let us consider the following differential equation:

$$
x^{\prime \prime}(t)+x^{\prime}(h(t))-x(h(t))=f(t), \quad t \in[0,1.6],
$$

with impulses (1.3), where $r=2$ and

$$
\begin{array}{lrl}
t_{1}=0.4, & \gamma_{1}=1.2, & \delta_{1}=0.7, \\
t_{2}=1, & \gamma_{2}=1.3, & \delta_{2}=0.95 .
\end{array}
$$

Let us assume that the deviation of the argument $h(t)$ has the following form:

$$
h(t)=t_{k}, \quad t \in\left[t_{k}, t_{k+1}\right), k=0,1,2 .
$$

According to [14], the Cauchy function of the first order impulsive equation

$$
y^{\prime}(t)+y(h(t))=0, \quad t \in[0,1.6],
$$

with impulses (1.3), where $r=2$ and

$$
\begin{array}{lr}
t_{1}=0.4, \quad \gamma_{1}=1.2, & \delta_{1}=0.7, \\
t_{2}=1, & \gamma_{2}=1.3, \quad \delta_{2}=0.95
\end{array}
$$

satisfies the inequality $C_{1}(t, s) \geq 0$, if

$$
\max _{k=1, \ldots, 3}\left(t_{k}-t_{k-1}\right)<1
$$

where $t_{0}=0, t_{3}=1.6$.

In our example, the condition (3.16) holds. Solving the homogeneous impulsive equation with the same left-hand side as in (3.11) with the initial conditions $x_{1}(0)=1, x_{1}^{\prime}(0)=0$ and $x_{2}(0)=0, x_{2}^{\prime}(0)=1$, we obtain

$$
\begin{gathered}
x_{1}(t)= \begin{cases}0, & t<0, \\
0.5 t^{2}+1, & t \in[0,0.4), \\
0.508(t-0.4)^{2}+0.28 t+1.184, & t \in[0.4,1), \\
0.648(t-1.0)^{2}+0.845 t+1.296, & t \in[1,1.6),\end{cases} \\
x_{2}(t)= \begin{cases}0, & t<0, \\
-0.5 t^{2}+t, & t \in[0,0.4), \\
-0.018(t-0.4)^{2}+0.42 t+0.216, & t \in[0.4,1), \\
0.22(t-1.0)^{2}+0.378 t+0.44, & t \in[1,1.6),\end{cases}
\end{gathered}
$$

see Fig. 2(a). 


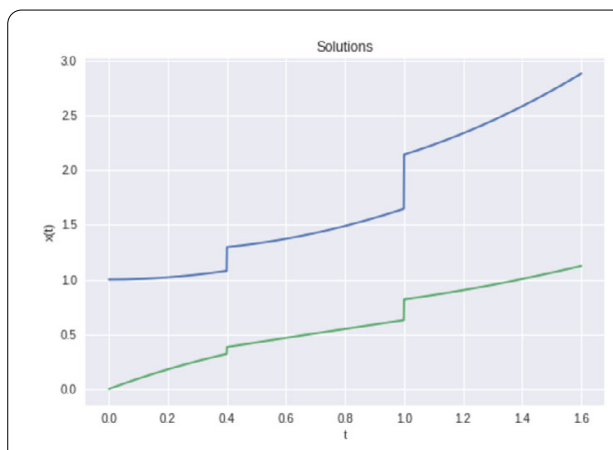

(a) Example of solutions

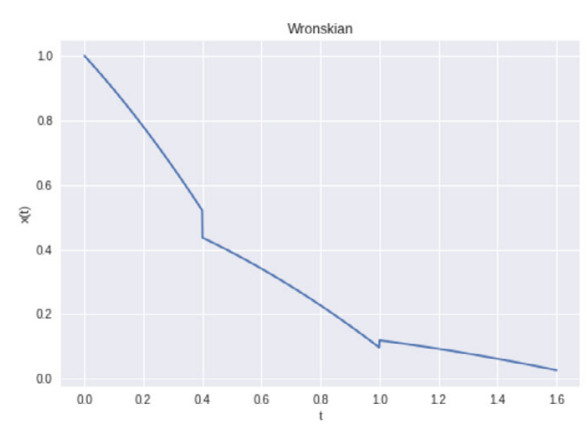

(b) Wronskian

Figure 2 Calculating the Wronskian of the fundamental system of solutions of a homogeneous equation

Calculating the Wronskian, we obtain

$$
W(t)= \begin{cases}0, & t<0, \\ -0.5 t^{2}-t+1, & t \in[0,0.4), \\ -0.218 t^{2}-0.262 t+0.577, & t \in[0.4,1), \\ -0.059 t^{2}+0.178, & t \in[1,1.6),\end{cases}
$$

see Fig. 2(b).

Thus, the conditions (1)-(3), (5) of Theorem 3.1 are fulfilled. According to Theorem 3.1, for each $A(t)$, satisfying the condition (3.5), the Green's function $G_{1}(t, s)$ is nonpositive.

\section{Sign-constancy of Green's function for nonlocal boundary value problem in the case when $b_{j}(t)$ can change sign}

For the case when $b_{j}(t)$ changes its sign, there can be considered an auxiliary impulsive differential equation:

$$
\begin{aligned}
& \left(L^{-} x\right)(t) \equiv x^{\prime \prime}(t)+\sum_{j=1}^{p} a_{j}(t) x^{\prime}\left(t-\tau_{j}(t)\right)+\sum_{j=1}^{p} b_{j}^{-}(t) x\left(t-\theta_{j}(t)\right)=z(t), \quad t \in[0, \omega], \\
& x\left(t_{k}\right)=\gamma_{k} x\left(t_{k}-0\right), \quad x^{\prime}\left(t_{k}\right)=\delta_{k} x^{\prime}\left(t_{k}-0\right), \quad k=1,2, \ldots, r, \\
& 0=t_{0}<t_{1}<t_{2}<\cdots<t_{r}<t_{r+1}=\omega \\
& x(\zeta)=0, \quad x^{\prime}(\zeta)=0, \quad \zeta<0
\end{aligned}
$$

where

$$
b_{j}^{-}(t)= \begin{cases}b_{j}(t), & b_{j}(t) \leq 0 \\ 0, & b_{j}(t)>0\end{cases}
$$

The solution for the boundary value problems (4.1)-(4.3), (3.2) can be written in the form

$$
x(t)=\left(G_{1}^{-} z\right)(t) \equiv \int_{0}^{\omega} G_{1}^{-}(t, s) z(s) d s .
$$


The given equation (1.2) can be written as

$$
(L x)(t)=\left(L^{-} x\right)(t)+\sum_{j=1}^{p} b_{j}^{+}(t) x\left(t-\theta_{j}(t)\right)=f(t)
$$

where

$$
b_{j}^{+}(t)= \begin{cases}b_{j}(t), & b_{j}(t)>0 \\ 0, & b_{j}(t) \leq 0\end{cases}
$$

After substituting (4.5) into (4.6) we obtain

$$
z(t)+\sum_{j=1}^{p} b_{j}^{+}(t) \int_{0}^{\omega} G_{1}^{-}\left(t-\theta_{j}(t), s\right) \chi_{[0, \omega]}\left(t-\theta_{j}(t)\right) z(s) d s=f(t)
$$

Define the integral operator $K: L_{\infty} \rightarrow L_{\infty}$ by the equality

$$
(K z)(t)=-\sum_{j=1}^{p} b_{j}^{+}(t) \int_{0}^{\omega}\left[G_{1}^{-}\left(t-\theta_{j}(t), s\right) \chi_{[0, \omega]}\left(t-\theta_{j}(t)\right)\right] z(s) d s
$$

Let us denote its spectral radius by $\rho(K)$.

We prove the assertion about the nonpositivity of the Green's function of (1.2)-(1.4), (3.2) without the assumption about the sign-constancy of $b_{j}(t)$.

Theorem 4.1 Assume that the following conditions are fulfilled:

(1) $a_{j}(t) \geq 0, j=1, \ldots, p, t \in[0, \omega]$.

(2) The Wronskian $W(t)$ of the fundamental system of solutions of a homogeneous equation (2.2)-(2.4) satisfies $W(t) \neq 0, t \in[0, \omega]$.

(3) The Cauchy function $C_{1}(t, s)$ of the first order equation (2.8) is positive for $0 \leq s \leq t \leq \omega$.

(4) $A(t)$ satisfies (3.5) for $t \in[0, \omega]$.

(5) $\gamma_{k} \geq 1, \delta_{k} \geq 0, k=1,2, \ldots, r$.

(6) The spectral radius $\rho(K)$ of the operator $K: L_{\infty} \rightarrow L_{\infty}$, defined by (4.9), satisfies the inequality $\rho(K)<1$.

Then Green's function $G_{1}(t, s)$ of the nonlocal problem (1.2)-(1.4), (3.2) is nonpositive for $t, s \in[0, \omega]$.

Proof The conditions (1)-(5) of Theorem 4.1 correspond to all the conditions of Theorem 3.1, so they imply that the Green's function $G_{1}^{-}(t, s)$ of the auxiliary boundary value problem (4.1)-(4.3), (3.2) is nonpositive for $t, s \in[0, \omega]$.

We have noted above, in Eq. (4.7), that $b_{j}^{+}(t) \geq 0$. Together with the fact that $G_{1}^{-}(t, s) \leq 0$, this implies that the operator $K$ is positive.

If the condition $\rho(K)<1$ holds, then Eq. (4.8) can be written as follows:

$$
z=(I-K)^{-1} f=\left[\sum_{j=0}^{\infty} K^{j}\right] f
$$


It follows from the inequality $G_{1}^{-}(t, s) \leq 0$ that all operators $K^{j}$ are positive and, consequently, for this case, the operator $\sum_{j=0}^{\infty} K^{j}$ is positive.

The solution $x(t)$ of the given boundary value problem (1.2)-(1.4), (3.2) can be written in the form

$$
x=\left(G_{1}^{-} \sum_{j=0}^{\infty} K^{j}\right) f,
$$

where the Green's operator for the problem (1.2)-(1.4), (3.2) can be presented in the form

$$
G_{1}=G_{1}^{-} \sum_{j=0}^{\infty} K^{j}
$$

The operator $G_{1}$ is nonpositive, if the condition $\rho(K)<1$ holds.

In order to verify the condition (6) we can use the results obtained in [13]. This condition is satisfied when the following inequality is fulfilled:

$$
\begin{aligned}
& \frac{\omega}{\prod_{k=1}^{r} \delta_{k}} \operatorname{ess} \sup \sum_{t \in[0, \omega]}^{p}\left|a_{j}(t)\right| \\
& \quad+\omega\left(\sum_{i=1}^{r} \frac{t_{i}-t_{i-1}}{\prod_{k=i}^{r} \delta_{k} \prod_{k=1}^{i} \gamma_{k}} \gamma_{i}+\frac{\omega-t_{r}}{\prod_{k=1}^{r} \gamma_{k}}\right) \underset{t \in[0, \omega]}{\operatorname{ess} \sup } \sum_{j=1}^{p}\left|b_{j}(t)\right|<1 .
\end{aligned}
$$

Example 4.1 Let us consider the following differential equation:

$$
x^{\prime \prime}(t)+0.2 x^{\prime}(h(t))-0.2(t-1) x(h(t))=f(t), \quad t \in[0,1.6]
$$

with impulses (1.3), where $r=2$ and

$$
\begin{aligned}
& t_{1}=0.4, \quad \gamma_{1}=1.2, \quad \delta_{1}=0.7, \\
& t_{2}=1, \quad \gamma_{2}=1.3, \quad \delta_{2}=0.95 \text {. }
\end{aligned}
$$

Let us assume that the deviation of the argument $h(t)$ has the following form:

$$
h(t)=t_{k}, \quad t \in\left[t_{k}, t_{k+1}\right), k=0,1,2 .
$$

According to [14], the Cauchy function of the first order impulsive equation

$$
y^{\prime}(t)+0.2 y(h(t))=0, \quad t \in[0,1.6],
$$

with impulses (1.3), where $r=2$ and

$$
\begin{aligned}
& t_{1}=0.4, \quad \gamma_{1}=1.2, \quad \delta_{1}=0.7, \\
& t_{2}=1, \quad \gamma_{2}=1.3, \quad \delta_{2}=0.95 \text {, }
\end{aligned}
$$




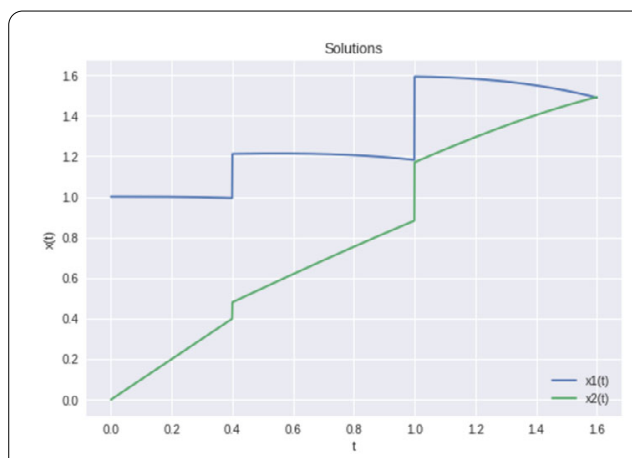

(a) Example of solutions

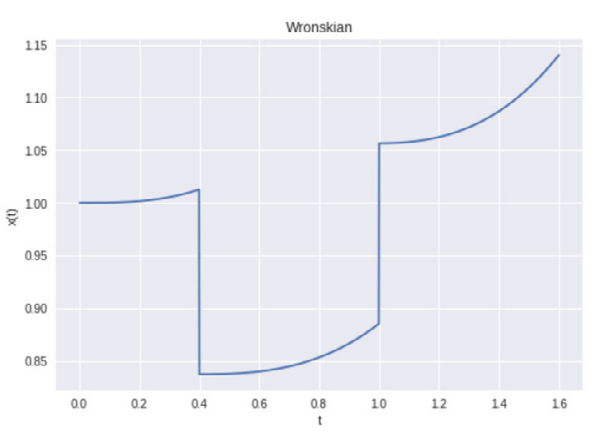

(b) Wronskian

Figure 3 Calculating the Wronskian of the fundamental system of solutions of a homogeneous equation

satisfies the inequality $C_{1}(t, s) \geq 0$, if

$$
0.2 \max _{k=1, \ldots, 3}\left(t_{k}-t_{k-1}\right)<1
$$

where $t_{0}=0, t_{3}=1.6$.

In our example, the condition (4.19) holds. Solving the homogeneous impulsive equation with the same left-hand side as in (4.14) with the initial conditions $x_{1}(0)=1, x_{1}^{\prime}(0)=0$ and $x_{2}(0)=0, x_{2}^{\prime}(0)=1$, we obtain

$$
\begin{aligned}
& x_{1}(t)= \begin{cases}0, & t<0, \\
-0.1 t^{3}+1, & t \in[0,0.4), \\
-0.121 t(t-0.4)^{2}+0.0224 t+1.203, & t \in[0.4,1), \\
-0.159 t(t-1.0)^{2}-0.0202 t+1.613, & t \in[1,1.6),\end{cases} \\
& x_{2}(t)= \begin{cases}0, & t<0, \\
t, & t \in[0,0.4), \\
-0.048 t(t-0.4)^{2}+0.7 t+0.2, & t \in[0.4,1), \\
-0.117 t(t-1.0)^{2}+0.649 t+0.521, & t \in[1,1.6),\end{cases}
\end{aligned}
$$

see Fig. 3(a).

Calculating the Wronskian, we obtain

$$
W(t)= \begin{cases}0, & t<0, \\ 0.2 t^{3}+1, & t \in[0,0.4), \\ 0.167 t^{3}-0.167 t^{2}+0.0536 t+0.832, & t \in[0.4,1), \\ 0.211 t^{3}-0.528 t^{2}+0.423 t+0.951, & t \in[1,1.6),\end{cases}
$$

see Fig. 3(b).

Let us verify, whether the condition $\rho(K)<1$ is satisfied. For our example, after substitution into (4.13), this inequality has the form $0.965<1$. Thus, the spectral radius $\rho(K)<1$. 
Thus, the conditions (1)-(3), (5)-(6) of Theorem 4.1 are fulfilled. According to Theorem 4.1, for each $A(t)$, satisfying the condition (3.5), the Green's function $G_{1}(t, s)$ is nonpositive.

\section{Acknowledgements}

This paper is part of the second author's Ph.D. thesis, which is being carried out in the Department of Mathematics at Ariel University.

\section{Funding}

Not applicable

Availability of data and materials

Not applicable.

\section{Competing interests}

The authors declare that they have no competing interests.

\section{Authors' contributions}

All authors contributed equally to the writing of this paper. All authors read and approved the final manuscript.

\section{Publisher's Note}

Springer Nature remains neutral with regard to jurisdictional claims in published maps and institutional affiliations.

\section{Received: 6 May 2019 Accepted: 28 October 2019 Published online: 06 November 2019}

\section{References}

1. Agarwal, R.P., Berezansky, L., Braverman, E., Domoshnitsky, A.: Nonoscillation Theory of Functional Differential Equations with Applications. Springer, New York (2012)

2. Agarwal, R.P., O'Regan, D.: Multiple nonnegative solutions for second order impulsive differential equations. Appl. Math. Comput. 114(1), 51-59 (2000)

3. Agarwal, R.P., O'Regan, D.: Upper, lower solutions for singular problems with nonlinear boundary data. NoDEA Nonlinear Differ. Equ. Appl. 9, 419-440 (2002)

4. Azbelev, N.V., Maksimov, V.P., Rakhmatullina, L.F.: Introduction to the Theory of Functional Differential Equations: Methods and Applications. Hindawi, New York (2007)

5. Baxley, J.: A singular nonlinear boundary value problem: membrane response of a spherical cap. SIAM J. Appl. Math. 48, 497-505 (1988)

6. Bolojan-Nica, O., Infante, G., Pietramala, P.: Existence results for impulsive systems with initial nonlocal conditions. Math. Model. Anal. 18(5), 599-611 (2013)

7. Bolojan-Nica, O., Infante, G., Precup, R.: Existence results for systems with coupled nonlocal initial conditions. Nonlinear Anal. 94, 231-242 (2014)

8. Calamai, A., Infante, G.: Nontrivial solutions of boundary value problems for second order functional differential equations. arXiv:1406.7508v1 [math. CA] (2014)

9. Domoshnitsky, A.: Maximum principles and nonoscillation intervals for first order Volterra functional differentia equations. Dyn. Contin. Discrete Impuls. Syst., Ser. A Math. Anal. 15, 769-814 (2008)

10. Domoshnitsky, A., Drakhlin, M.: Nonoscillation of first order impulsive differential equations with delay. J. Math. Anal. Appl. 206(1), 254-269 (1997)

11. Domoshnitsky, A., Landsman, G.: Semi-nonoscillation intervals in the analysis of sign constancy of Green's functions of Dirichlet, Neumann and focal impulsive problems. Adv. Differ. Equ. 2017, 81 (2017)

12. Domoshnitsky, A., Landsman, G., Yanetz, S.: About sign-constancy of Green's functions of one-point problem for impulsive second order delay equations. Funct. Differ. Equ. 21(1-2), 3-15 (2014)

13. Domoshnitsky, A., Mizgireva, lu.: Sign-constancy of Green's functions for two-point impulsive boundary value problems. Miskolc Math. Notes 20, 193-208 (2019)

14. Domoshnitsky, A., Mizgireva, lu., Raichik, V.: Semi-nonoscillation intervals and sign-constancy of Green's functions of two-point impulsive boundary value problems. Ukr. Math. J. (accepted) (2019)

15. Domoshnitsky, A., Volinsky, I.: About positivity of Green's functions for nonlocal boundary value problems with impulsive delay equations. Sci. World J. 2014, Article ID 978519 (2014)

16. Domoshnitsky, A., Volinsky, l.: About differential inequalities for nonlocal boundary value problems with impulsive delay equations. Math. Bohem. 140(2), 121-128 (2015)

17. Domoshnitsky, A., Volinsky, I., Shklyar, R.: About Green's functions for impulsive differential equations. Funct. Differ. Equ. 20, 55-81 (2013)

18. Feller, W.: Diffusion processes in one dimension. Trans. Am. Math. Soc. 77, 1-31 (1954)

19. Feng, M., Xie, D.: Multiple positive solutions of multi-point boundary value problem for second-order impulsive differential equations. J. Math. Anal. Appl. 223(1), 438-448 (2009)

20. Graef, J.R., Henderson, J., Yang, B.: Existence and nonexistence of positive solutions of an n-th order nonlocal boundary value problem. Proc. Dyn. Syst. Appl. 5, 86-191 (2007)

21. Graef, J.R., Yang, B.: Positive solutions of a third order nonlocal boundary value problem. Discrete Contin. Dyn. Syst., Ser. S 1, 89-97 (2008)

22. Guidotti, P., Merino, S.: Gradial loss of positivity and hidden invariant cones in a scalar heat equation. Differ. Integral Equ. 13, 1551-1568 (2000) 
23. Hakl, R., Lomtatidze, A., Sremr, J.: Some boundary value problems for first order scalar functional differential equations. FOLIA, Masaryk University, Brno, Czech Republic (2002)

24. Hu, L., Liu, L., Wu, Y.: Positive solutions of nonlinear singular two-point boundary value problems for second-order impulsive differential equations. Nonlinear Anal., Theory Methods Appl. 69(11), 3774-3789 (2008)

25. Infante, G.: Positive solutions of some nonlinear BVPs involving singularities and integral BCs. Discrete Contin. Dyn. Syst., Ser. S 1(1), 99-106 (2008)

26. Infante, G., Minhos, F., Pietramala, P.: Non-negative solutions of systems of ODEs with coupled boundary conditions. Commun. Nonlinear Sci. Numer. Simul. 17(12), 4952-4960 (2012)

27. Infante, G., Webb, J.: Nonlinear non-local boundary-value problems and perturbed Hammerstein integral equations. Proc. Edinb. Math. Soc. 49(3), 637-656 (2006)

28. Jankowsky, T.: Positive solutions to second order four-point boundary value problems for impulsive differential equations. Appl. Math. Comput. 202(2), 550-561 (2008)

29. Jiang, D., Lin, X.: Multiple positive solutions of Dirichlet boundary value problems for second order impulsive differential equations. J. Math. Anal. Appl. 321(2), 501-514 (2006)

30. Karaca, I.Y.: On positive solutions for second-order boundary value problems of functional differential equations. Appl. Math. Comput. 219, 5433-5439 (2013)

31. Karakostas, I.Y., Mavridis, K.G., Tsamatos, P.Ch.: Triple solutions for a nonlocal functional boundary value problem by Leggett-Williams theorem. Appl. Anal. 83, 957-970 (2004)

32. Karakostas, I.Y., Tsamatos, P.Ch.: Existence of multiple positive solutions for a nonlocal boundary value problem. Topol. Methods Nonlinear Anal. 19, 109-121 (2002)

33. Kiguradze, I., Puza, B.: On boundary value problems for systems of linear functional differential equations. Czechoslov Math. J. 47(2), 341-373 (1997)

34. Kiguradze, I., Puza, B.: Boundary value problems for systems of linear functional differential equations. FOLIA, Brno, Czech Republic (2002)

35. Lakshmikantham, V., Bainov, D.D., Simeonov, P.S.: Theory of Impulsive Differential Equations. World Scientific, Singapore (1989)

36. Lee, E.K., Lee, Y.H.: Multiple positive solutions of singular two point boundary value problems for second order impulsive differential equations. Appl. Math. Comput. 158(3), 745-759 (2004)

37. Ma, R.: A survey on nonlocal boundary value problems. Appl. Math. E-Notes 7, 257-279 (2001)

38. Mizgireva, lu.: On positivity of Green's functions of two-point impulsive problems. Funct. Differ. Equ. 25(3-4), 189-200 (2018)

39. Ntouyas, S.K.: Nonlocal Initial and Boundary Value Problems: A Survey, Handbook of Differential Equations: Ordinary Differential Equations, Volume II. Elsevier, Amsterdam (2005)

40. O'Regan, D.: Upper and lower solutions for singular problems arising in the theory of membrane response of a spherical cap. Nonlinear Anal. 47, 1163-1174 (2001)

41. Pandit, S.G., Deo, S.G.: Differential Systems Involving Impulses. Springer, Berlin (1982)

42. Picone, M.: Su un problema al contorno nelle equazioni differenziali lineari ordinarie del secondo ordine. Ann. Sc Norm. Super. Pisa, Cl. Sci. 10, 1-95 (1908)

43. Precup, R., Trif, D.: Multiple positive solutions of non-local initial value problems for first order differential systems. Nonlinear Anal. 75, 5961-5970 (2012)

44. Samoilenko, A.M., Perestyuk, N.A.: Impulsive Differential Equations. World Scientific, Singapore (1995)

45. Skubachevskii, A.L.: Elliptic Functional Differential Equations and Applications. Birkhäuser, Basel (1997)

46. Skubachevskii, A.L.: Nonclassical boundary value problems. J. Math. Sci. 155(2), 199-334 (2008)

47. Sommerfeld, S.: Ein beitrag zur hydrodynamiscen Erklarung der turbulenten Flussigkeitsbewegungen. In: Atti del IV Congr. Intern dei Matem. Rome, vol. 3, pp. 116-124 (1909)

48. Webb, J.: Multiple positive solutions of some nonlinear heat flow problems. Discrete Contin. Dyn. Syst. 2005(suppl.), 895-903 (2005)

49. Webb, J.: Optimal constants in a nonlocal boundary value problem. Nonlinear Anal. 63, 672-685 (2005)

50. Webb, J., Infante, G.: Positive solutions of nonlocal boundary value problems: a unified approach. J. Lond. Math. Soc. $74,673-693(2006)$

51. Webb, J., Infante, G.: Positive solutions of nonlocal boundary value problems involving integral conditions. NoDEA Nonlinear Differ. Equ. Appl. 15, 45-67 (2008)

52. Whyburn, W.M.: Differential equations with general boundary conditions. Bull. Am. Math. Soc. 48, $692-704$ (1942)

53. Yang, Z:: Positive solutions of a second-order integral boundary value problem. J. Math. Anal. Appl. 321, 751-765 (2006)

54. Zavalishchin, S.G., Sesekin, A.N.: Dynamic Impulse Systems: Theory and Applications. Springer, Dordrecht (1997) 